\title{
Differences in rituximab use between pediatric rheumatologists and nephrologists for the treatment of refractory lupus nephritis and renal flare in childhood-onset SLE
}

\author{
Mileka Gilbert ${ }^{1 *}$ (D, Beatrice Goilav², Joyce J. Hsu ${ }^{3}$, Paul J. Nietert ${ }^{1}$, Esra Meidan ${ }^{4}$, Annabelle Chua ${ }^{5}$, Stacy P. Ardoin ${ }^{6}$, \\ Scott E. Wenderfer ${ }^{7}$, Emily von Scheven ${ }^{8}$, Natasha M. Ruth ${ }^{1}$ and for the Pediatric Nephrology and Rheumatology \\ Collaborative Group, the Childhood Arthritis and Rheumatology Research Alliance, and the American Society for \\ Pediatric Nephrology
}

\begin{abstract}
Background: Consensus treatment plans have been developed for induction therapy of newly diagnosed proliferative lupus nephritis (LN) in childhood-onset systemic lupus erythematosus. However, patients who do not respond to initial therapy, or who develop renal flare after remission, warrant escalation of treatment. Our objective was to assess current practices of pediatric nephrologists and rheumatologists in North America in treatment of refractory proliferative $L N$ and flare.

Methods: Members of Childhood Arthritis and Rheumatology Research Alliance (CARRA) and the American Society for Pediatric Nephrology (ASPN) were surveyed in November 2015 to assess therapy choices (other than modifying steroid dosing) and level of agreement between rheumatologists and nephrologists for proliferative LN patients. Two cases were presented: (1) refractory disease after induction treatment with corticosteroid and cyclophosphamide (CYC) and (2) nephritis flare after initial response to treatment. Survey respondents chose treatments for three follow up scenarios for each case that varied by severity of presentation. Treatment options included CYC, mycophenolate mofetil (MMF), rituximab (RTX), and others, alone or in combination.

Results: Seventy-six respondents from ASPN and foty-one respondents from CARRA represented approximately $15 \%$ of the eligible members from each organization. Treatment choices between nephrologists and rheumatologists were highly variable and received greater than $50 \%$ agreement for an individual treatment choice in only the following 2 of 6 follow up scenarios: $59 \%$ of nephrologists, but only $38 \%$ of rheumatologists, chose increasing dose of MMF in the case of LN refractory to induction therapy with proteinuria, hematuria, and improved serum creatinine. In a follow up scenario showing severe renal flare after achieving remission with induction
\end{abstract}

\footnotetext{
* Correspondence: gilbertm@musc.edu

'Medical University of South Carolina, 135 Rutledge Ave, MSC 561, Charleston, SC 29425, USA

Full list of author information is available at the end of the article
} 
therapy, $58 \%$ of rheumatologists chose CYC and RTX combination therapy, whereas the top choice for nephrologists (43\%) was CYC alone. Rheumatologists in comparison to nephrologists chose more therapy options that contained RTX in all follow up scenarios except one $(p<0.05)$.

Conclusions: Therapy choices for pediatric rheumatologists and nephrologists in the treatment of refractory LN or $L N$ flare were highly variable with rheumatologists more often choosing rituximab. Further investigation is necessary to delineate the reasons behind this finding. This study highlights the importance of collaborative efforts in developing consensus treatment plans for pediatric LN.

Keywords: Juvenile systemic lupus erythematosus, Refractory lupus nephritis, Renal flare, Therapy choices, Pediatric rheumatologist, Pediatric nephrologist

\section{Background}

Systemic lupus erythematosus (SLE) is an autoimmunemediated disease that can cause inflammation of multiple organ systems. The involvement of the kidneys, which occurs in over half of childhood SLE, significantly alters morbidity and mortality and therefore requires more aggressive immunosuppression [1, 2]. A meta-analysis showed 10-30 \% higher prevalence of LN in childhood-onset SLE compared to adult-onset SLE [3], with up to $70 \%$ of children with SLE having nephritis compared to about $50 \%$ of adults with lupus $[4,5]$. In the 1980 and 1990s, standardof-care treatment with IV cyclophosphamide (CYC) was delineated by the National Institutes of Health for adults with proliferative LN [6-9]. More recently, mycophenolate mofetil (MMF) was found by the Aspreva Lupus Management Study investigators to be non-inferior to IV CYC [10], and both drugs are now used first-line for the treatment of proliferative LN. More black and Hispanic patients notably responded to MMF versus IV CYC [10].

While childhood-onset SLE comprises only about 15$20 \%$ of all lupus patients [4,5], it is difficult to perform large randomized controlled trials (RCTs) in pediatric LN given small numbers. The Childhood Arthritis and Rheumatology Research Alliance (CARRA) has developed consensus treatment plans (CTPs) in an effort to reduce heterogeneity in treatment and better enable future comparative effectiveness studies. CTPs for the induction treatment of proliferative LN were developed in 2012, and treatment arms include both IV CYC and MMF [11]. As in adults, current first-line treatment for initial diagnosis of proliferative LN encompasses CYC or MMF, along with the use of corticosteroids.

However, despite advances in the treatment of proliferative LN, a substantial number of patients never reach complete or even partial renal response after induction and are diagnosed as treatment refractory nonresponders (NR). Eighteen to $69 \%$ of adult patients with LN were resistant to CYC with variable definitions of treatment response $[8,12-15]$, whereas $5-48 \%$ of patients treated with MMF were described as NR [15-17]. Likewise, at 6 and 12 months, $40-66 \%$ and $65-75 \%$ of children with proliferative LN achieved remission [18-22].

In the patients who do reach complete or partial renal response, unfortunately, renal flares can occur in about $50 \%$ of adult-onset SLE after long term follow up [23, 24]. In children with SLE, renal flares can occur in $25-50 \%$ of patients on therapy $[25,26]$. Each subsequent flare leads to more kidney injury and heightened risk for kidney failure.

Patients who do not adequately respond to the CTP regimens, or who develop disease flare after remission, warrant change in treatment. Clinical guidance is lacking for this particularly vulnerable pediatric population, as there are no RCTs or CTPs for cases of NR/refractory LN. Several case series from single centers and case reports describe the use of other medications for treating refractory LN, including biologic agents that target $\mathrm{B}$ cell (rituximab, ofatumumab, belimumab) and $\mathrm{T}$ cell activity (abatacept, calcineurin inhibitors cyclosporine and tacrolimus) [27, 28]. Given the involvement of B cells in the pathogenesis of LN, rituximab (RTX) is one of the most widely employed rescue medications for patients who do not respond to standard treatment or in patients who flare [29].

Depending on the geographic location of a patient and availability of subspecialists in close proximity, pediatric nephrologists may be the primary subspecialists caring for patients with LN. Conversely, pediatric rheumatologists may be treating patients with $\mathrm{LN}$ as the leading organ system involvement in a SLE patient. Given this difficult-totreat disease, treatment practices may vary by type of subspecialist, treating center, and even within practices.

In this study, members from both CARRA and the American Society for Pediatric Nephrology (ASPN) were surveyed to determine the current opinions and practices in the treatment of proliferative LN refractory to induction therapy and in patients who have a renal flare after achieving renal response in childhood-onset SLE.

\section{Methods}

A web-based survey to assess immunosuppression treatment choices and level of agreement between pediatric rheumatologists and nephrologists for pediatric patients 
with proliferative LN was developed by the Pediatric Nephrology and Rheumatology Collaborative Group (PNR-CG) comprised of members of CARRA and the ASPN. Internal Review Board exemption for the study was obtained through Nationwide Children's Hospital. The survey was sent to the membership of CARRA and the ASPN via Survey Monkey in November 2015. There were 76 respondents from ASPN and 41 respondents from CARRA representing approximately $15 \%$ of the eligible members from each organization.

Two cases were presented in the survey: (1) Pediatric patient with proliferative LN refractory to 6 months of induction therapy with CYC (See Additional File 1) and (2) Pediatric patient with renal flare 3 months after achieving remission in response to induction therapy for proliferative LN (See Additional File 2).

Renal response and flare definitions defined in CARRA proliferative LN CTPs [11] were used in the cases. In summary, the core renal parameters were proteinuria, renal function, and urine sediment. Substantial response (complete remission) was defined as normalization of renal function, inactive urine sediment, plus spot protein/creatinine ratio $<0.2$. Moderate and mild renal responses were defined as at least $50 \%$ improvement (moderate) or $30-50 \%$ improvement (mild) in two core renal parameters without clinically relevant worsening of the remaining core parameter. NR included any patient who did not qualify for mild, moderate, or substantial response. Proteinuric/nephrotic renal flare was defined as a persistent increase in urine protein/creatinine ratios to values $>0.5$ after achieving complete response, or a doubling of proteinuria with values $>1.0$ after achieving a partial response. Non-proteinuric/nephritic renal flare was defined as increase or recurrence of active urinary sediment with or without increase in proteinuria.

Physicians were surveyed on treatment choices for three fictional follow up scenarios for each case. The treatment choices included IV CYC, MMF, calcineurin inhibitors, RTX, belimumab, RTX in combination with IV CYC, and RTX in combination with MMF. Statistical analyses of differences between responses of pediatric nephrologists and rheumatologists were determined using Fisher's Exact Test with $p$ value $<0.05$ (two-sided) considered statistically significant.

\section{Results}

The survey was distributed to members of the ASPN and CARRA who completed fellowship programs in pediatric nephrology and pediatric rheumatology. The 76 respondents from ASPN and 41 respondents from CARRA represented approximately 15\% of the eligible members from each organization. All respondents were board-eligible or board-certified in their subspecialty with the vast majority having at least 2 years of post- fellowship experience, and $45 \%$ of pediatric nephrologists and $60 \%$ of pediatric rheumatologists having greater than 10 years of experience. Fifty-two percent of pediatric nephrologists reported managing less than 25 patients with SLE, and half of pediatric rheumatologists reported managing 25 to 100 patients with SLE (Table 1). Only $51 \%$ of the pediatric nephrologists and $24 \%$ of the pediatric rheumatologists surveyed in this study follow a standard protocol for treatment of LN (Table 1).

For the case of refractory class IV LN (See Additional File 1), a 16-year-old female failed induction therapy with 7 monthly doses of CYC $500-1000 \mathrm{mg} / \mathrm{m}^{2}$ IV in addition to steroids. Although fever, rash, and arthritis improved, a repeat renal biopsy showed persistent activity, little chronicity, and no evidence of membranous LN. In the first follow-up scenario (See Additional File 1), patient was found to have no renal response to induction therapy with hypertension, peripheral edema, hypocomplementemia, hypoalbuminemia, positive dsDNA antibody, persistent proteinuria, and active urine sediment, as well as a significant increase in serum creatinine. Survey respondents were asked to consider their next choice in immunosuppressive agent. Pediatric nephrologists and rheumatologists agreed with the top choice of MMF in combination with RTX ( $42 \%$ vs. $44 \%$, Fig. 1). CYC in combination with RTX (16\% vs. $27 \%$, Fig. 1 ) and MMF alone ( $20 \%$ vs. $20 \%$, Fig. 1) were the next most common choices for therapy. Overall, while the choices varied within groups, there was no difference between nephrologist and rheumatologist responses $(p=0.40)$.

In the second follow-up scenario of refractory class IV LN after CYC induction therapy (See Additional File 1), the patient was found to have mild renal response with hypertension, peripheral edema, hypocomplementemia, hypoalbuminemia, positive dsDNA antibody, and persistent proteinuria, but improvement in serum creatinine and only mild hematuria. Pediatric nephrologists and rheumatologists chose MMF alone (41\% vs. $37 \%$, Fig. 2 ) and MMF in combination with RTX ( $15 \%$ vs. $34 \%$, Fig. 2) as their top choices for next step in immunosuppressive therapy in this scenario. Overall, there was no difference between nephrologist and rheumatologist responses $(p=0.10)$. However, the majority of rheumatologists $(53 \%)$ chose therapies that involved RTX alone or in combination, compared to only $31 \%$ of nephrologists that chose RTX therapies (Fig. 2). There was a difference between the groups of nephrologist and rheumatologist responses in choices of RTX-containing regimens versus choices without RTX $(p=0.03)$.

In a third follow-up scenario of the refractory class IV LN case (See Additional File 1), the patient was found to have moderate renal response with persistent hypertension, 
Table 1 Demographics of survey respondents

\begin{tabular}{|c|c|c|}
\hline & Pediatric nephrologists & Pediatric rheumatologists \\
\hline Total Respondents & $N=76^{\mathrm{a}}$ & $N=41^{a}$ \\
\hline Medical Centers & $N=56$ & $N=15$ \\
\hline Peds Neph Board-Eligible & $100 \%$ & $0 \%$ \\
\hline Peds Rheum Board-Eligible & $0 \%$ & $100 \%$ \\
\hline \multicolumn{3}{|l|}{ Years in Practice } \\
\hline$<2$ & $2 \%$ & $7 \%$ \\
\hline $2-5$ & $29 \%$ & $17 \%$ \\
\hline $6-10$ & $25 \%$ & $17 \%$ \\
\hline$>10$ & $45 \%$ & $60 \%$ \\
\hline \multicolumn{3}{|l|}{ \# of Pediatric SLE Patients } \\
\hline $0-25$ & $52 \%$ & $37 \%$ \\
\hline $25-50$ & $14 \%$ & $20 \%$ \\
\hline $50-100$ & $19 \%$ & $30 \%$ \\
\hline$>100$ & $6 \%$ & $13 \%$ \\
\hline LN Standard Protocol & $51 \%$ & $24 \%$ \\
\hline
\end{tabular}

${ }^{a}$ Approximately $15 \%$ of ASPN and CARRA membership

resolution of peripheral edema, hypocomplementemia, hypoalbuminemia, positive dsDNA antibody, active urine sediment, but greater than $50 \%$ improvement in serum creatinine and proteinuria. Whereas a majority of pediatric nephrologists chose MMF alone (59\%, Fig. 3), rheumatologists were split over use of MMF alone (38\%) or in combination with RTX (35\%) as their top choices for next step in immunosuppressive therapy. Overall, there was a difference between nephrologist and rheumatologist responses $(p<$ 0.01 ), unlike in the prior follow-up scenarios. Additionally, rheumatologists again chose more therapies that involved RTX compared to nephrologists ( $59 \%$ vs. $21 \%, p<0.01$, Fig. 3). Thus, pediatric rheumatologists were more likely to use RTX in this scenario of active urine sediment but

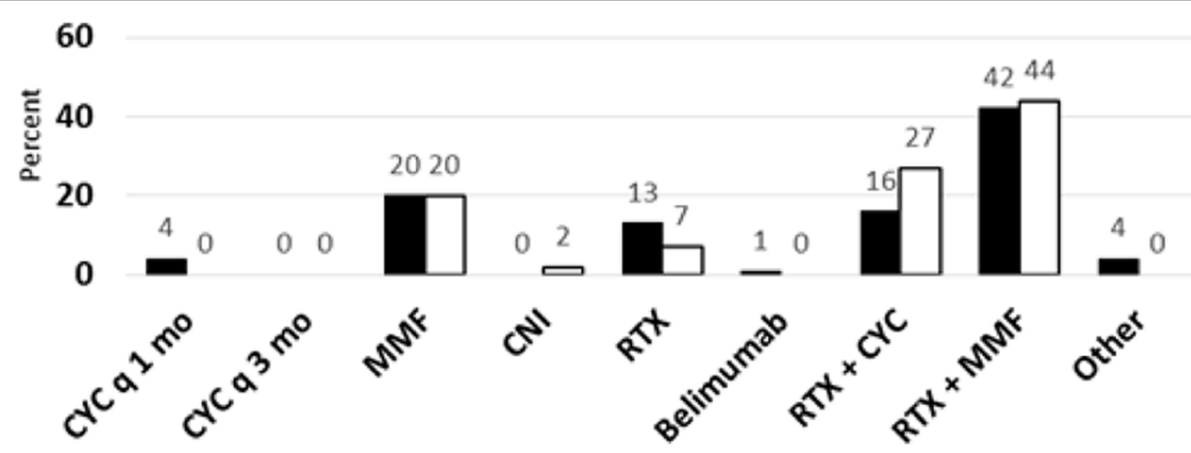

Nephrologists $\square$ Rheumatologists

\begin{tabular}{|l|c|c|c|}
\hline & Nephrologists & Rheumatologists & $\boldsymbol{p}$ value \\
\hline All choices & & & 0.40 \\
\hline RTX vs. Non-RTX & $71 \%$ vs. $29 \%$ & $78 \%$ vs. $22 \%$ & 0.51 \\
\hline CYC vs. MMF vs. Other & $20 \%$ vs. $62 \%$ vs. $18 \%$ & $27 \%$ vs. $63 \%$ vs. $10 \%$ & 0.41 \\
\hline
\end{tabular}

Fig. 1 Treatment choices for LN refractory to induction therapy with renal insufficiency, nephrotic syndrome, and hematuria. Seventy-six nephrologists and forty-one rheumatologists responded to survey question. Treatment choices are depicted in graph. Statistical analysis of differences in responses between groups is depicted in chart. $P$ value $<0.05$ is considered statistically significant. (RTX $=$ Rituximab, CNI $=$ Calcineurin Inhibitor, MMF = Mycophenolate mofetil, CYC = Cyclophosphamide) 


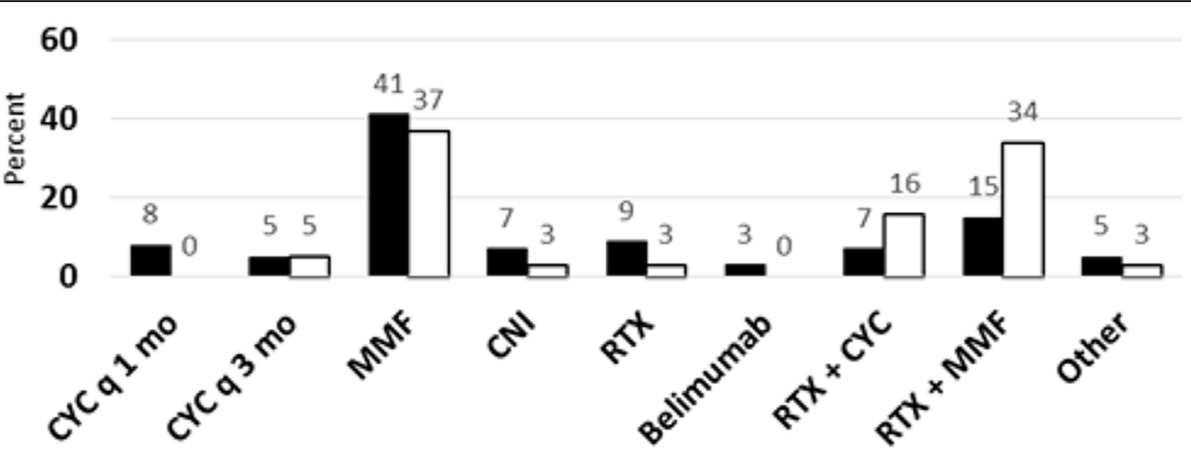

- Nephrologists $\quad$ Rheumatologists

\begin{tabular}{|l|c|c|c|}
\hline & Nephrologists & Rheumatologists & $\boldsymbol{p}$ value \\
\hline All choices & & & 0.10 \\
\hline RTX vs. Non-RTX & $31 \%$ vs. $69 \%$ & $53 \%$ vs. $47 \%$ & 0.03 \\
\hline CYC vs. MMF vs. Other & $20 \%$ vs. $56 \%$ vs. $24 \%$ & $21 \%$ vs. $71 \%$ vs. $8 \%$ & 0.10 \\
\hline
\end{tabular}

Fig. 2 Treatment choices for $L N$ refractory to induction therapy with nephrotic syndrome, improved hematuria and creatinine. Seventy-five nephrologists and thirty-eight rheumatologists responded to survey question. Treatment choices are depicted in graph. Statistical analysis of differences in responses between groups is depicted in chart. $P$ value $<0.05$ is considered statistically significant. (RTX $=$ Rituximab, CNI $=$ Calcineurin Inhibitor, MMF = Mycophenolate mofetil, CYC = Cyclophosphamide)

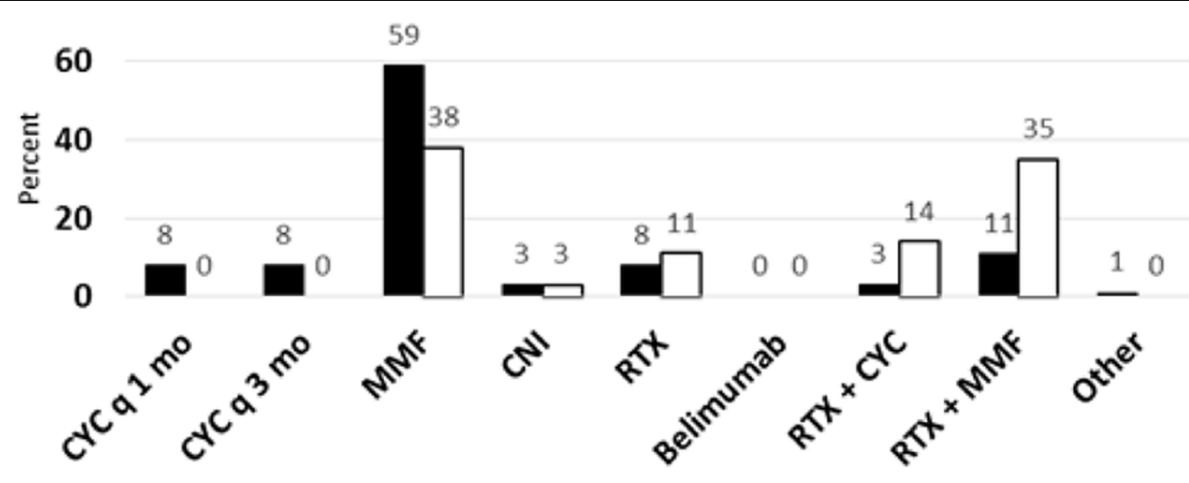

- Nephrologists $\square$ Rheumatologists

\begin{tabular}{|l|c|c|c|}
\hline & Nephrologists & Rheumatologists & $p$ value \\
\hline All choices & & & $<0.01$ \\
\hline RTX vs. Non-RTX & $21 \%$ vs. $79 \%$ & $59 \%$ vs. $41 \%$ & $<0.01$ \\
\hline CYC vs. MMF vs. Other & $19 \%$ vs. $69 \%$ vs. $12 \%$ & $14 \%$ vs. $73 \%$ vs. $14 \%$ & 0.81 \\
\hline
\end{tabular}

Fig. 3 Treatment choices for LN refractory to induction therapy with hematuria, improved creatinine and proteinuria. Seventy-five nephrologists and thirty-seven rheumatologists responded to survey question. Treatment choices are depicted in graph. Statistical analysis of differences in responses between groups is depicted in table. $P$ value $<0.05$ is considered statistically significant. (RTX = Rituximab, CNI $=$ Calcineurin Inhibitor, $\mathrm{MMF}=$ Mycophenolate mofetil, $\mathrm{CYC}=$ Cyclophosphamide) 
improvement in other renal parameters, and thus may be more aggressive with use of RTX. In all follow-up scenarios, there was no difference between groups of nephrologist and rheumatologist choices in therapies when considering additional CYC versus MMF or other options.

The second clinical vignette presented a case of a 12year-old patient with SLE and class IV LN who achieved complete renal remission after induction therapy with CYC in addition to steroids for proliferative LN according to published CTP [11] (See Additional File 2). She was transitioned to MMF $1000 \mathrm{mg}$ twice daily for maintenance therapy and tapering prednisone dose. Three months later, she developed nephrotic syndrome, hematuria with active urine sediment, hypocomplementemia, and high titer dsDNA antibody, without change in serum creatinine. She was diagnosed with renal flare, and repeat renal biopsy showed class IV LN with high activity and low chronicity scores. Pediatric nephrologists chose CYC alone (22\%) or increased dose of MMF to $1500 \mathrm{mg}$ twice daily (22\%), whereas pediatric rheumatologists chose CYC in combination with RTX (36\%) or CYC alone $(21 \%)$ as their top choices for therapy (Fig. 4).

If the second case used MMF as the induction agent instead of CYC at diagnosis (See Additional File 2), nephrologists chose increased dose of MMF to $1500 \mathrm{mg}$ twice daily $(39 \%)$ or CYC alone $(25 \%)$ to treat renal flare (Fig. 5). Rheumatologists chose CYC alone (27\%) or MMF in combination with RTX (24\%) as their top choices for treatment of renal flare (Fig. 5).

In the third follow up scenario of renal flare after complete renal remission was achieved with $\mathrm{CYC}$ and steroids followed by maintenance MMF therapy, the patient was also found to have elevated serum creatinine, rapidly progressive glomerulonephritis, and class IV LN on repeat renal biopsy (See Additional File 2). Nephrologists and rheumatologists ( $81 \%$ vs. $82 \%$, Fig. 6) agreed that CYC with or without RTX were the best therapeutic choices for renal flare in this scenario, although more rheumatologists compared to nephrologists (52\% vs. $22 \%$, Fig. 6) would use CYC in combination with RTX.

In all three follow up scenarios involving case of renal flare after achieving remission with induction therapy, there were differences noted between nephrologists and rheumatologists $(p=0.02,0.02,0.04$ respectively; Figs. 4 , 5 and 6). Additionally, rheumatologists chose more therapies that involved RTX than nephrologists in all scenarios ( $p=0.03,0.02,<0.01$ respectively; Figs. 4, 5 and 6). There was no difference between groups of nephrologist and rheumatologist choices in therapies when considering additional CYC versus MMF or other options. Thus, pediatric rheumatologists chose RTX in more situations of renal flare than pediatric nephrologists, similar to the first case of proliferative LN refractory to induction

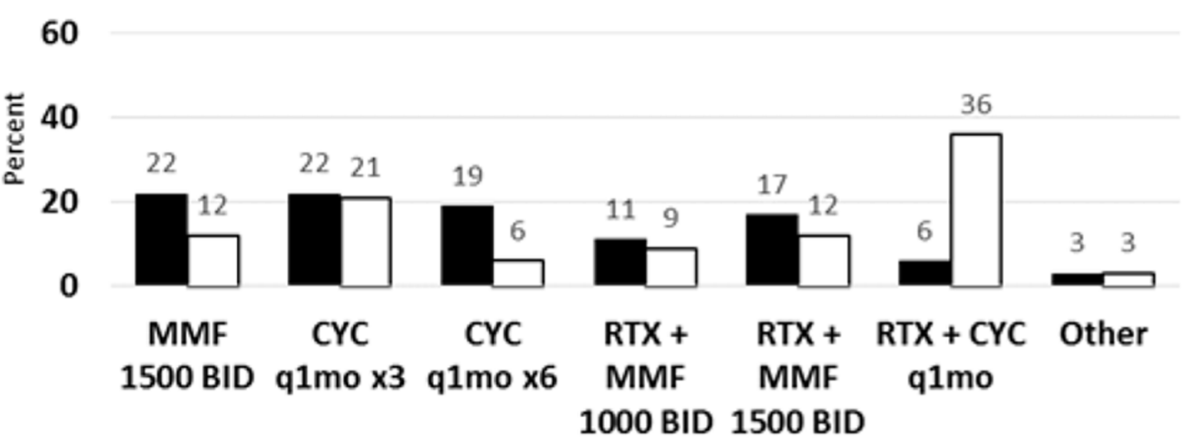

- Nephrologists $\square$ Rheumatologists

\begin{tabular}{|l|c|c|c|}
\hline & Nephrologists & Rheumatologists & $p$ value \\
\hline All choices & & & 0.02 \\
\hline RTX vs. Non-RTX & $34 \%$ vs. $66 \%$ & $58 \%$ vs. $42 \%$ & 0.03 \\
\hline CYC vs. MMF vs. Other & $47 \%$ vs. $50 \%$ vs. $3 \%$ & $64 \%$ vs. $33 \%$ vs. $3 \%$ & 0.24 \\
\hline
\end{tabular}

Fig. 4 Treatment choices for renal flare in proliferative LN after achieving remission with CYC induction therapy. Sixty-four nephrologists and thirty-three rheumatologists responded to survey question. Treatment choices are depicted in graph. Statistical analysis of differences in responses between groups is depicted in chart. $P$ value $<0.05$ is considered statistically significant. (RTX = Rituximab, CNI =Calcineurin Inhibitor, MMF = Mycophenolate mofetil, $C Y C=$ Cyclophosphamide) 


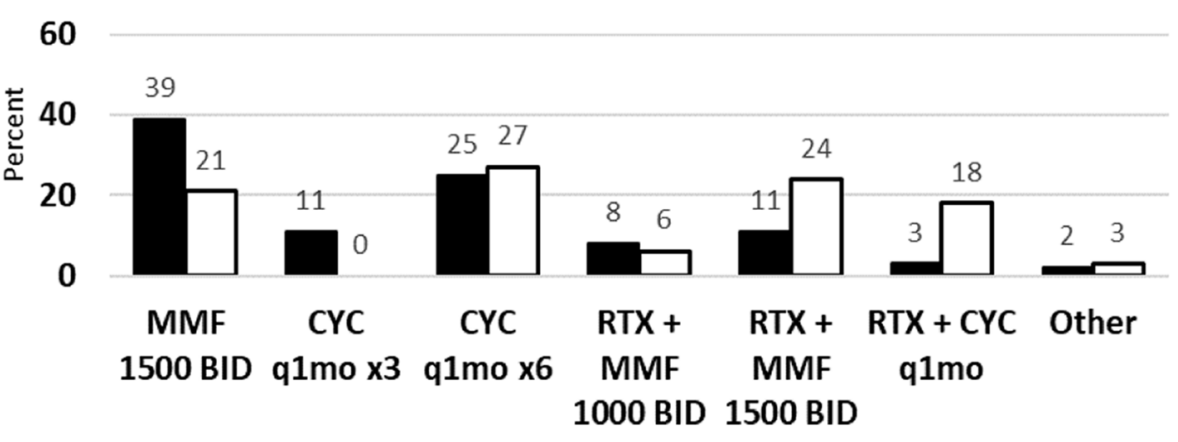

Nephrologists aRheumatologists

\begin{tabular}{|l|c|c|c|}
\hline & Nephrologists & Rheumatologists & $\boldsymbol{p}$ value \\
\hline All choices & & & 0.02 \\
\hline RTX vs. Non-RTX & $23 \%$ vs. $77 \%$ & $48 \%$ vs. $52 \%$ & 0.02 \\
\hline CYC vs. MMF vs. Other & $39 \%$ vs. $59 \%$ vs. $2 \%$ & $45 \%$ vs. $52 \%$ vs. $3 \%$ & 0.70 \\
\hline
\end{tabular}

Fig. 5 Treatment choices for renal flare in proliferative LN after achieving remission with MMF induction therapy. Sixty-one nephrologists and thirty-three rheumatologists responded to survey question. Treatment choices are depicted in graph. Statistical analysis of differences in responses between groups is depicted in table. $P$ value $<0.05$ is considered statistically significant. (RTX = Rituximab, CNI $=C$ alcineurin Inhibitor, $\mathrm{MMF}=$ Mycophenolate mofetil, $\mathrm{CYC}=$ (yclophosphamide)

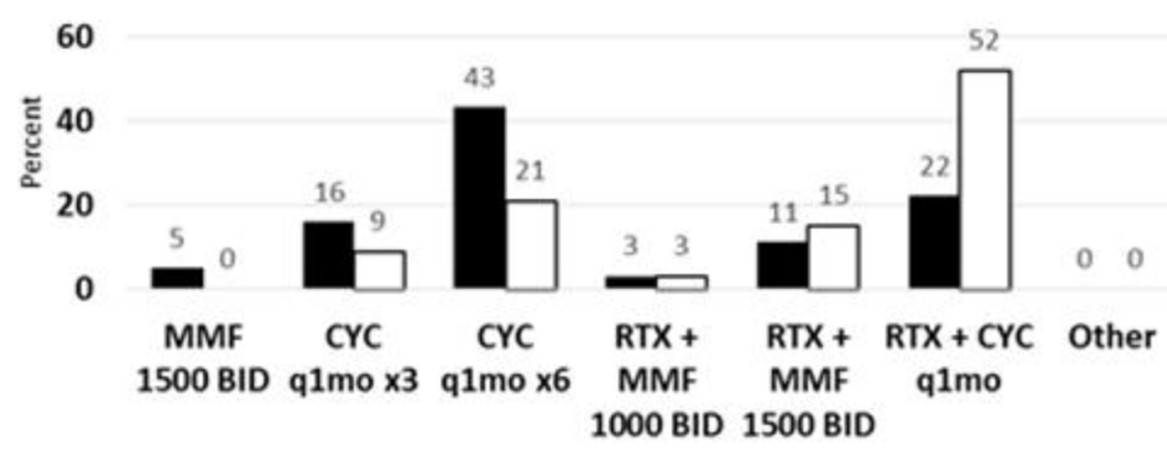

Nephrologists aRheumatologists

\begin{tabular}{|l|c|c|c|}
\hline & Nephrologists & Rheumatologists & p value \\
\hline All choices & & & 0.04 \\
\hline RTX vs. Non-RTX & $37 \%$ vs. $63 \%$ & $70 \%$ vs. $30 \%$ & $<0.01$ \\
\hline CYC vs. MMF vs. Other & $81 \%$ vs. $19 \%$ vs. $0 \%$ & $82 \%$ vs. $18 \%$ vs. $0 \%$ & 1.00 \\
\hline
\end{tabular}

Fig. 6 Treatment choices for renal flare after induction with renal insufficiency and rapidly progressive glomerulonephritis. Sixty-three nephrologists and thirty-three rheumatologists responded to survey question. Treatment choices are depicted in graph. Statistical analysis of differences in responses between groups is depicted in table. $P$ value $<0.05$ is considered statistically significant. (RTX $=$ Rituximab, CNI $=$ Calcineurin Inhibitor, MMF = Mycophenolate mofetil, CYC = Cyclophosphamide) 
therapy. Belimumab was included as a treatment option in both cases, however, was rarely chosen. Calcineurin inhibitors were also used in some patients by both rheumatology and nephrology and may have a role as well in resistant disease.

\section{Discussion}

The approach to refractory proliferative $\mathrm{LN}$ after initial therapy with CYC or MMF is limited by the absence of evidence-based studies. This is particularly true in the case of pediatric LN where there is a lack of clinical trials. Thus, pediatric rheumatologists and nephrologists must make decisions on treatment of refractory LN based on retrospective cohort studies.

In this study, there are limitations regarding the definitions chosen for complete remission and flare. For instance, substantial response (complete remission) was defined as normalization of renal function, inactive urine sediment, plus spot protein/creatinine ratio $<0.2$ consistent with the CARRA CTPs for the induction treatment of proliferative LN [11]. Recently, the adult LN community has changed the complete response definition to be far more liberal, with a spot protein/creatinine ratio $<0.5$ and in more recent literature $<0.7$ is now acceptable.

CTPs are a useful tool to compare effectiveness of currently practiced treatments in the clinical setting. The first step in developing CTPs for refractory proliferative $\mathrm{LN}$ in pediatric patients is to understand the clinical practices of pediatric rheumatologists and nephrologists.

This survey assessed the choices of immunosuppressive agents in clinical scenarios of refractory and relapsing childhood-onset proliferative LN. Treatment choices between nephrologists and rheumatologists were highly variable. Of note, pediatric rheumatologists and nephrologists did agree on the treatment of the most severe case of proliferative LN that was refractory to induction therapy, and both groups tended to choose more aggressive treatment options. However, there were differences in choice of therapies in the more moderate cases of refractory proliferative $\mathrm{LN}$ and in all cases of renal flare, particularly in the use of RTX.

While RTX in combination with MMF and corticosteroids did not meet primary endpoint of renal response in $\mathrm{LN}$ in patients $16-75$ years of age after 1 year of treatment in the randomized, double-blind, placebocontrolled LUNAR clinical trial [30], small prospective studies of use of RTX alone or in combination with MMF for induction therapy of childhood LN have shown some efficacy and steroid-sparing effect $[31,32]$. Hogan et al. looked at 12 patients with $\mathrm{LN}$ and found therapy with RTX + MMF combined with a rapid decrease in steroid appears to be an efficacious treatment for severe LN but was unfortunately associated with three varicella-zoster virus infections. Basu et al. assessed 44 patients with active $\mathrm{LN}$ and found that flarefree survival was significantly higher at 36 months with RTX compared with MMF and CYC (100\% for RTX vs. $83 \%$ for MMF and $53 \%$ for CYC, $\mathrm{p}=0.006$ ) [31]. Requirement of mean daily dosage of prednisone was significantly lower in RTX group at 36 months compared with other groups (RTX vs. MMF, $p=0.005$; RTX vs. CYC, $p=0.0001$ ) [32]. The degree of circulating B cell depletion by RTX appears to be critical for clinical effect and may explain the LUNAR trial results [33]. Metaanalyses of RTX treatment in refractory LN support an additional benefit [34, 35]. Moreover, several case series in childhood-onset LN report complete and partial remission at varying rates and steroid-sparing effect of RTX in refractory cases [29]. EULAR [36], ACR [37], and Kidney Disease Improving Global Outcomes (nonprofit organization developing and implementing evidence-based clinical practice guidelines) [38, 39] guidelines support the use of RTX as one of the treatment options in patients with refractory LN.

It is unclear as to why pediatric rheumatologists may treat more aggressively given rheumatologist's increased choice of RTX in more moderate case scenarios of refractory and relapsing LN in this survey; however, it may be that more nephrologists reported using a standardized protocol. Unfortunately, this survey did not ask a question regarding which standardized protocol (KDIGO or adapt the ACR or EULAR guidelines for children). Pediatric rheumatologists may also be more comfortable with using RTX as this medication is also used for other rheumatologic indications with renal and non-renal manifestations; however, nephrologists also use rituximab for other nephrotic syndromes. This study highlights the importance of collaborative effort in developing CTPs for pediatric LN, as there are significant differences in the management of LN between pediatric rheumatologists and nephrologists.

Given the lack of FDA (Food and Drug Administration) approved therapies for $\mathrm{LN}$ and lack of guidelines for refractory LN, there are several biases and limitations of this case-based survey. Familiarity with treatment options with use for other indications may affect choice of therapy. Access to certain medications may be limited by insurance and thus affect physician experience with certain medications like belimumab. Belimumab was FDA approved for pediatric SLE in 2019 and recently has been shown as an effective add-on therapy for induction therapy for proliferative LN [40-42], but was not in widespread use at the time of this survey in 2015 . The approach for nephrologists and rheumatologists is likely evolving now that the results of the BLISS-LN (Efficacy and Safety of Belimumab in Patients with Active LN) trial have been published [40]. Fifteen of pediatric nephrologists and rheumatologists in the ASPN and 
CARRA responded to the cases in the survey likely reflecting a sampling bias for those physicians who take care of more patients with SLE; although the majority of nephrologists surveyed manage less than 25 pediatric patients with LN. The low response rate to the survey is certainly a limitation of this study and may not be representative of the practice of these specialists in the United States and does not include any participants from Europe or other countries; however, the vast majority of pediatric rheumatologists and $49 \%$ of pediatric nephrologists surveyed do not follow a standard protocol for treatment of LN and are not following CARRA CTPs. Thus, even with higher response rates, we would likely still see highly variable responses to the clinical scenarios presented. In the future, the study could be improved by sending repeat reminder emails to participants and by finding a way to incentivize participants who complete the survey. Another limitation to the study is the delay between the survey being sent to the participants (2015) and the publication. New drugs for SLE are not rapidly coming to market but it would be interesting to see if survey responses have changed in 2021 especially with Belimumab getting approved for the treatment of LN.

\section{Conclusions}

Therapy choices for pediatric rheumatologists and nephrologists in the treatment of proliferative LN refractory to induction therapy or LN flare after remission were highly variable. In addition, there were differences between rheumatologists and nephrologists particularly in the use of RTX for the majority of scenarios presented in these cases. This provides an opportunity to work towards consensus to reduce heterogeneity in the treatment of refractory pediatric LN so that we can perform comparative effectiveness trials. This study highlights the importance of collaborative effort in developing CTPs for pediatric $\mathrm{LN}$.

\section{Abbreviations}

ACR: American College of Rheumatology; ASPN: American Society of Pediatric Nephrology; CARRA: Childhood Arthritis and Rheumatology Research Alliance; CTP: Consensus Treatment Plan; CYC: Cyclophosphamide; DNA: Deoxyribonucleic acid; EULAR: European League Against Rheumatism; FDA: Food and Drug Administration; ISN/RPS: International Society of Nephrology and the Renal Pathology Society; KDIGO: Kidney Disease Improving Global Outcomes; LN: Lupus nephritis; LUNAR: Lupus Nephritis Assessment with Rituximab study; MMF: Mycophenolate mofetil; NR: Nonresponders; PNR-CG: Pediatric Nephrology and Rheumatology Collaborative Group; RCT: Randomized controlled trial; RTX: Rituximab; SLE: Systemic lupus erythematosus

\section{Supplementary Information}

The online version contains supplementary material available at https://doi. org/10.1186/s12969-021-00627-w.

Additional file 1.

Additional file 2 .

\section{Acknowledgements}

The authors would like to acknowledge the ASPN and CARRA organizations for distributing the survey to their members. The authors wish to acknowledge CARRA and the ongoing Arthritis Foundation financial support of CARRA.

\section{Authors' contributions}

$M G, B G$, and $J J H$ participated in development of survey, interpretation of results, and were major contributors in writing the manuscript. PJN performed statistical analysis and participated in interpretation of results. EM, AC, and SPA participated in development of survey. SW and EVS participated in development of survey and interpretation of results. NMR participated in interpretation of results and was a major contributor in writing the manuscript. All authors read and approved the final manuscript.

\section{Authors' information}

Not applicable.

\section{Funding}

There was no direct funding for this study, but PJN's effort on this project was funded by several NIH grants (NCATS grant \# UL1-TR001450, NIAMS grant \# P30-AR072582, NIGMS grant \# U54-GM104941).

\section{Availability of data and materials}

All data generated or analyzed during this study are included in this manuscript.

\section{Declarations}

Ethics approval and consent to participate

Internal Review Board exemption for study was obtained at Nationwide Children's Hospital, IRB \# IRB15-00843.

\section{Consent for publication}

Not applicable.

\section{Competing interests}

The authors have no financial competing interests.

\section{Author details}

'Medical University of South Carolina, 135 Rutledge Ave, MSC 561, Charleston, SC 29425, USA. ${ }^{2}$ The Children's Hospital at Montefiore, 111 East 210th Street, Bronx, NY 10467, USA. ${ }^{3}$ Stanford University School of Medicine, 291 Campus Drive, Stanford, CA 94305, USA. ${ }^{4}$ Harvard Medical School, 300 Longwood Ave, Boston, MA 02115, USA. ${ }^{5}$ Duke University, Box 3959, 2301 Erwin Road, Durham, NC 27710, USA. ${ }^{6}$ Nationwide Children's Hospital, 700 Children's Drive, Columbus, OH 43221, USA. 'Baylor College of Medicine, One Baylor Plaza, Houston, TX 77030, USA. ${ }^{8}$ University of California San Francisco, 550 16th Street, 4th floor, San Francisco, CA 94158, USA.

Received: 31 March 2021 Accepted: 24 July 2021

Published online: 30 August 2021

\section{References}

1. Cervera R, Khamashta MA, Font J, Sebastiani GD, Gil A, Lavilla P, et al. Morbidity and mortality in systemic lupus erythematosus during a 10-year period: a comparison of early and late manifestations in a cohort of 1,000 patients. Med (Baltim). 2003;82(5):299-308.

2. Oni L, Wright RD, Marks S, Beresford MW, Tullus K. Kidney outcomes for children with lupus nephritis. Pediatr Nephrol. 2021;36:1377-85.

3. Livingston B, Bonner A, Pope J. Differences in clinical manifestations between childhood-onset lupus and adult-onset lupus: a meta-analysis. Lupus. 2011;20(13):1345-55.

4. Thakur N, Rai N, Batra P. Pediatric Lupus Nephritis-Review of Literature. Curr Rheumatol Rev. 2017;13(1):29-36.

5. Couture J, Silverman ED. Update on the pathogenesis and treatment of childhood-onset systemic lupus erythematosus. Curr Opin Rheumatol. 2016; 28(5):488-96.

6. Austin HA 3rd, Klippel JH, Balow JE, le Riche NG, Steinberg AD, Plotz PH, et al. Therapy of lupus nephritis. Controlled trial of prednisone and cytotoxic drugs. N Engl J Med. 1986;314(10):614-9. 
7. Boumpas DT, Austin HA 3rd, Vaughn EM, Klippel JH, Steinberg AD, Yarboro $\mathrm{CH}$, et al. Controlled trial of pulse methylprednisolone versus two regimens of pulse cyclophosphamide in severe lupus nephritis. Lancet. 1992; 340(8822):741-5

8. Gourley MF, Austin HA 3rd, Scott D, Yarboro CH, Vaughan EM, Muir J, et al. Methylprednisolone and cyclophosphamide, alone or in combination, in patients with lupus nephritis. A randomized, controlled trial. Ann Intern Med. 1996;125(7):549-57.

9. Illei GG, Austin HA, Crane M, Collins L, Gourley MF, Yarboro CH, et al. Combination therapy with pulse cyclophosphamide plus pulse methylprednisolone improves long-term renal outcome without adding toxicity in patients with lupus nephritis. Ann Intern Med. 2001;135(4):248-57.

10. Appel GB, Contreras G, Dooley MA, Ginzler EM, Isenberg D, Jayne D, et al. Mycophenolate mofetil versus cyclophosphamide for induction treatment of lupus nephritis. J Am Soc Nephrol. 2009;20(5):1103-12.

11. Mina R, von Scheven E, Ardoin SP, Eberhard BA, Punaro M, llowite N, et al. Consensus treatment plans for induction therapy of newly diagnosed proliferative lupus nephritis in juvenile systemic lupus erythematosus. Arthritis Care Res (Hoboken). 2012;64(3):375-83.

12. Korbet SM, Lewis EJ, Schwartz MM, Reichlin M, Evans J, Rohde RD. Factors predictive of outcome in severe lupus nephritis. Lupus Nephritis Collaborative Study Group. Am J Kidney Dis. 2000;35(5):904-14.

13. Ioannidis JP, Boki KA, Katsorida ME, Drosos AA, Skopouli FN, Boletis JN, et al. Remission, relapse, and re-remission of proliferative lupus nephritis treated with cyclophosphamide. Kidney Int. 2000;57(1):258-64.

14. Mok CC, Ying KY, Tang S, Leung CY, Lee KW, Ng WL, et al. Predictors and outcome of renal flares after successful cyclophosphamide treatment for diffuse proliferative lupus glomerulonephritis. Arthritis Rheum. 2004;50(8):2559-68.

15. Ginzler EM, Dooley MA, Aranow C, Kim MY, Buyon J, Merrill JT, et al. Mycophenolate mofetil or intravenous cyclophosphamide for lupus nephritis. N Engl J Med. 2005;353(21):2219-28.

16. Chan TM, Li FK, Tang CS, Wong RW, Fang GX, Ji YL, et al. Efficacy of mycophenolate mofetil in patients with diffuse proliferative lupus nephritis. Hong Kong-Guangzhou Nephrology Study Group. N Engl J Med. 2000; 343(16):1156-62

17. Hu W, Liu Z, Chen H, Tang Z, Wang Q, Shen K, et al. Mycophenolate mofetil vs cyclophosphamide therapy for patients with diffuse proliferative lupus nephritis. Chin Med J (Engl). 2002;115(5):705-9.

18. Rianthavorn P, Buddhasri A. Long-term renal outcomes of childhood-onset global and segmental diffuse proliferative lupus nephritis. Pediatr Nephrol. 2015;30(11):1969-76.

19. Askenazi D, Myones B, Kamdar A, Warren R, Perez M, De Guzman M, et al. Outcomes of children with proliferative lupus nephritis: the role of protocol renal biopsy. Pediatr Nephrol. 2007;22(7):981-6.

20. Lee BS, Cho HY, Kim EJ, Kang HG, Ha IS, Cheong HI, et al. Clinical outcomes of childhood lupus nephritis: a single center's experience. Pediatr Nephrol. 2007;22(2):222-31.

21. Boneparth A, Ilowite NT, Investigators CR. Comparison of renal response parameters for juvenile membranous plus proliferative lupus nephritis versus isolated proliferative lupus nephritis: a cross-sectional analysis of the CARRA Registry. Lupus. 2014;23(9):898-904.

22. Smith E, Al-Abadi E, Armon K, Bailey K, Ciurtin C, Davidson J, et al. Outcomes following mycophenolate mofetil versus cyclophosphamide induction treatment for proliferative juvenile-onset lupus nephritis. Lupus. 2019;28(5):613-20.

23. Moroni G, Gallelli B, Quaglini S, Banfi G, Rivolta E, Messa P, et al. Withdrawal of therapy in patients with proliferative lupus nephritis: long-term follow-up. Nephrol Dial Transplant. 2006;21(6):1541-8.

24. Illei GG, Takada K, Parkin D, Austin HA, Crane M, Yarboro CH, et al. Renal flares are common in patients with severe proliferative lupus nephritis treated with pulse immunosuppressive therapy: long-term followup of a cohort of 145 patients participating in randomized controlled studies. Arthritis Rheum. 2002;46(4):995-1002.

25. Wu JY, Yeh KW, Huang JL. Early predictors of outcomes in pediatric lupus nephritis: focus on proliferative lesions. Semin Arthritis Rheum. 2014;43(4):513-20.

26. Aragon $\mathrm{E}$, Resontoc LP, Chan $Y H$, Lau YW, Tan PH, Loh HL, et al. Long-term outcomes with multi-targeted immunosuppressive protocol in children with severe proliferative lupus nephritis. Lupus. 2016;25(4):399-406.

27. Kronbichler A, Brezina B, Gauckler P, Quintana LF, Jayne DRW. Refractory lupus nephritis: When, why and how to treat. Autoimmun Rev. 2019;18(5): 510-8.
28. Group AT. Treatment of lupus nephritis with abatacept: the Abatacept and Cyclophosphamide Combination Efficacy and Safety Study. Arthritis Rheumatol. 2014;66(11):3096-104.

29. Mahmoud I, Jellouli M, Boukhris I, Charfi R, Ben Tekaya A, Saidane O, et al. Efficacy and safety of rituximab in the management of pediatric systemic lupus erythematosus: a systematic review. J Pediatr. 2017;187:213-9.

30. Rovin BH, Furie R, Latinis K, Looney RJ, Fervenza FC, Sanchez-Guerrero J, et al. Efficacy and safety of rituximab in patients with active proliferative lupus nephritis: the Lupus Nephritis Assessment with Rituximab study. Arthritis Rheum. 2012;64(4):1215-26.

31. Hogan J, Godron A, Baudouin V, Kwon T, Harambat J, Deschenes G, et al. Combination therapy of rituximab and mycophenolate mofetil in childhood lupus nephritis. Pediatr Nephrol. 2018;33(1):111-6.

32. Basu B, Roy B, Babu BG. Efficacy and safety of rituximab in comparison with common induction therapies in pediatric active lupus nephritis. Pediatr Nephrol. 2017;32(6):1013-21.

33. Gomez Mendez LM, Cascino MD, Garg J, Katsumoto TR, Brakeman P, Dall'Era M, et al. Peripheral Blood B Cell Depletion after Rituximab and Complete Response in Lupus Nephritis. Clin J Am Soc Nephrol. 2018;13(10):1502-9.

34. Shamliyan TA, Dospinescu P. Additional Improvements in Clinical Response From Adjuvant Biologic Response Modifiers in Adults With Moderate to Severe Systemic Lupus Erythematosus Despite Immunosuppressive Agents: A Systematic Review and Meta-analysis. Clin Ther. 2017;39(7):1479-506. e45.

35. Alshaiki F, Obaid E, Almuallim A, Taha R, El-Haddad H, Almoallim H. Outcomes of rituximab therapy in refractory lupus: A meta-analysis. Eur J Rheumatol. 2018:5(2):118-26.

36. Bertsias GK, Tektonidou M, Amoura Z, Aringer M, Bajema I, Berden JH, et al. Joint European League Against Rheumatism and European Renal Association-European Dialysis and Transplant Association (EULAR/ERA-EDTA) recommendations for the management of adult and paediatric lupus nephritis. Ann Rheum Dis. 2012;71(11):1771-82.

37. Hahn BH, McMahon MA, Wilkinson A, Wallace WD, Daikh DI, Fitzgerald JD, et al. American College of Rheumatology guidelines for screening, treatment, and management of lupus nephritis. Arthritis Care Res (Hoboken). 2012;64(6):797-808.

38. Radhakrishnan J, Cattran DC. The KDIGO practice guideline on glomerulonephritis: reading between the (guide)lines-application to the individual patient. Kidney Int. 2012;82(8):840-56.

39. Rovin BH, Caster DJ, Cattran DC, Gibson KL, Hogan JJ, Moeller MJ, et al. Management and treatment of glomerular diseases (part 2): conclusions from a Kidney Disease: Improving Global Outcomes (KDIGO) Controversies Conference. Kidney Int. 2019;95(2):281-95.

40. Furie R, Rovin BH, Houssiau F, Malvar A, Teng YKO, Contreras G, et al. TwoYear, Randomized, Controlled Trial of Belimumab in Lupus Nephritis. N Engl J Med. 2020;383(12):1117-28.

41. Margiotta DPE, Basta F, Batani V, Afeltra A. Belimumab and low-doses of mycophenolate mofetil as induction therapy of class IV lupus nephritis: case series and literature review. BMC Nephrol. 2018;19(1):54.

42. Trentin F, Gatto M, Zen M, Larosa M, Maddalena L, Nalotto L, et al. Effectiveness, tolerability, and safety of belimumab in patients with refractory SLE: a review of observational clinical-practice-based studies. Clin Rev Allergy Immunol. 2018;54(2):331-43.

\section{Publisher's Note}

Springer Nature remains neutral with regard to jurisdictional claims in published maps and institutional affiliations.

Ready to submit your research? Choose BMC and benefit from:

- fast, convenient online submission

- thorough peer review by experienced researchers in your field

- rapid publication on acceptance

- support for research data, including large and complex data types

- gold Open Access which fosters wider collaboration and increased citations

- maximum visibility for your research: over $100 \mathrm{M}$ website views per year

At BMC, research is always in progress.

Learn more biomedcentral.com/submission 